\title{
Chiral Discrimination in Optical Binding
}

\author{
Kayn A. Forbes and David L. Andrews ${ }^{1}$ \\ School of Chemistry, University of East Anglia, Norwich NR4 7TJ, United Kingdom
}

\begin{abstract}
The laser-induced intermolecular force that exists between two or more particles in the presence of an electromagnetic field is commonly termed 'optical binding'. Distinct from the single-particle forces that are at play in optical trapping at the molecular level, the phenomenon of optical binding is a manifestation of the coupling between optically induced dipole moments in neutral particles. In other, more widely known areas of optics, there are many examples of chiral discrimination - signifying the different response a chiral material has to the handedness of an optical input. In the present analysis, extending previous work on chiral discrimination in optical binding, a new mechanism is identified using a quantum electrodynamical approach. It is shown that the optical binding force between a pair of chiral molecules can be significantly discriminatory in nature, depending upon both the handedness of the interacting particles and the polarization of the incident light, and it is typically several orders of magnitude larger than previously reported.
\end{abstract}

\section{INTRODUCTION}

Electromagnetic fields have a general capacity to produce inter-particle forces in matter, by the generation and coupling of static and dynamic polarizations. In the absence of any applied optical source, vacuum field fluctuations induce electric dipoles, whose coupling gives rise to the well-known Casimir-Polder potential.[1] Indeed it transpires even the well-known London or Hamaker[2] interactions, which occur between electrically neutral particles, can fundamentally be attributed to vacuum electromagnetic fields. However, when light is introduced to a material system, the associated fields can produce another kind of force. One increasingly prominent example, first predicted using quantum electrodynamics (QED)[3], is an energy shift generated by the light-induced polarization in non-polar interacting particles. This coupling between optically induced dipoles leads to an intermolecular force that is commonly referred to as optical binding $[4,5]$ (although it is not necessarily attractive in nature). A decade after the original QED analysis, Burns et al. [6] provided the first experimental studies of optical binding using light propagating orthogonally to the inter-particle displacement vector. In their pioneering studies, they made use of the then recently discovered phenomenon of optical tweezers[7] to isolate a pair of micron-sized polystyrene spheres, observing the radiation-induced interaction with respect to time. Their semiclassical analysis of the results concluded that the polystyrene spheres formed a bound structure with preferred inter-particle separations. Their first graphs of binding energy against particle separation exhibited rolling potential energy maxima and minima, and optical binding accordingly gained recognition[8] as a potentially very significant method for fabricating and manipulating micro- and nanoparticle arrays.

In the succeeding years many theoretical and experimental investigations followed, including several further quantum electrodynamical studies. These more recent studies have underscored the fact that optical binding forces offer a large number of distinctive attributes which can be used in the optical manipulation

\footnotetext{
${ }^{1}$ david.andrews@physics.org
} 
of matter. Two such examples of key experimental studies of optical binding occurred in 2002-2003, where two groups $[9,10]$ independently observed what is now termed "longitudinal optical binding". In their studies, using a counterpropagating dual beam trap, multiple particles entering the trap were observed to take up equilibrium positions along the propagation axes, with inter-particle separations several times the particle diameters. This work prompted a renewed attention to optical binding, and the longitudinal form has become the more widely studied system.[11-17] In a further development of theory, based on the adoption of advanced QED methods, results were then obtained in a form applicable to radiation fields with arbitrary polarization and beam geometry, delivering further physical insights into the fundamental mechanisms in operation.[18-20] One specific instance concerns particles of cylindrical symmetry such as carbon nanotubes (CNT). It was shown that for a typical single-walled CNT pair, the optically induced forces range between $10^{-5} \mathrm{~N}$ and $10^{-12}$, depending on geometry.[21]

More generally, the intricate local fields and forces produced by optical binding have been secured and illustrated through further QED analyses, generating richly structured potential energy landscapes.[22] These multidimensional landscapes pictorially represent the optically induced interactions between particles in the form of contour maps, on which the position and magnitude of local energy minima and maxima depend on the particle configuration and the polarization and wave-vector of the propagating light. Such maps can be produced for a wide range of multi-particle and beam geometries.[23-25] The analysis of local energy minima in these landscapes reveals points of stability and possible particle configurations for optical binding; other features such as local forces and torques between particles can also be readily derived. Subsequent QED studies have addressed the optical binding forces between molecular aggregates[23]; the effects broadband radiation[26] and secondary laser light[27], and light conveying orbital angular momentum[28, 29] (so-called structured light).[30] Whilst theory for both micro- and nanoparticles is now well developed, and a plethora of experiments demonstrating the phenomenon for micro-sized objects are well documented, only recently has optical binding been experimentally observed for nanoparticles[31,32] where, amongst other technical challenges, the more disruptive influence of thermal motions has to be overcome.

In the present study, our focus is on the novel effects that arise when the trapped particles are chiral. Optical forces and processes between chiral particles are generally dependent on the handedness of each material component and/or that of an applied circularly polarised beam: they are discriminatory.[33-35] It is worth emphasizing that optical processes occur when the initial and final states of the system (matter and radiation field) differ, with discriminatory examples including circular dichroism[36] and circular differential scattering.[37] In contrast, an optical force arises when both the initial and final states are identical, such as where discriminatory effects have recently been shown to occur in optical trapping.[38, 39] The discriminatory effect in chiral entities can be attributed to their low symmetry, where selection rules allow at least some electronic transitions to include contributions from not only electric dipole, but also other electric and magnetic multipole moments. Although small in comparison to electric-dipole forces, the leading term dependent on the handedness of each molecule typically arises from electric-magnetic dipole couplings, and these are the source of the chiral discrimination. Some chirality-dependent aspects of optical binding have previously been identified by QED analysis[40], in which it was found that for chiral molecules freely tumbling in the presence of circularly polarised light, the pair energy shift is independent of polarization and the discriminatory effects arise solely from the handedness of the molecules. The discriminatory effect was also estimated to be in the order of $10^{-6}$ smaller than the purely electric-electric dipole coupling forces. In the following analysis it is shown that there are in fact additional terms that contribute more significantly to the chiral discrimination in optical binding, typically several orders of magnitude larger than those previously reported. 


\section{THEORETICAL METHOD}

In non-relativistic QED, the Power-Zienau-Woolley Hamiltonian[41-43] is used to describe a closed system consisting of particles and electromagnetic fields. This Hamiltonian, equation (1) below, is written as a sum of three terms: (i) the second-quantized Hamiltonian for the radiation field in vacuo, $H_{\text {rad }}$; (ii) a sum over all particles with corresponding non-relativistic Hamiltonians, $H_{\text {mol }}(\xi)$; and (iii) a sum of the interaction Hamiltonians that describe the coupling between each particle and the electromagnetic field, $H_{\text {int }}(\xi)$ :

$$
H=H_{\mathrm{rad}}+\sum_{\xi} H_{\mathrm{mol}}(\xi)+\sum_{\xi} H_{\mathrm{int}}(\xi)
$$

The subscript 'mol' in the second term designates a common application to molecules; the general formalism is equally applicable to larger, electrically neutral, particles - as discussed later in this paper. In the following, for simplicity we first discuss and derive expressions for optical binding energies on the basis of interactions between individual molecules, for which the electronic transitions relate to the entirety of the particle. The last of the three terms $H_{\text {int }}(\xi)$ in the above equation, when expressed in the multipolar formalism[44] appears as a sum of multipolar contributions as given here (where the convention of implied summation of repeated indices is used);

$$
\begin{aligned}
H_{\mathrm{int}}(\xi) & =-\varepsilon_{0}^{-1} \mu_{i}(\xi) d_{i}^{\perp}\left(\mathbf{R}_{\xi}\right)-m_{i}(\xi) b_{i}\left(\mathbf{R}_{\xi}\right) \\
& -\varepsilon_{0}^{-1} Q_{i j}(\xi) \nabla_{j} d_{i}^{\perp}\left(\mathbf{R}_{\xi}\right)-\ldots,
\end{aligned}
$$

where $\mu_{i}(\xi), m_{i}(\xi)$, and $Q_{i j}(\xi)$ are electric-dipole, magnetic-dipole, and electric-quadrupole tensor operators, respectively. The first term in equation (2), which accounts for electric-dipole interactions, often suffices when describing a wide range of phenomena, including almost all previous studies of optical binding. The usually argued reason for this is that when the radiative wavelengths involved in the system are much larger than that of the particle's dimensions, the higher order spatial variations of the optical vector potential are of zero-order. In the canonical transformation that links interactions cast in terms of vector potential to the more familiar form engaging electric and magnetic multipoles, it is the terms involving spatial variation of the vector potential that correlate with magnetic dipole, electric quadrupole and higher electric and magnetic multipoles.[45] In the multipolar formalism the interaction Hamiltonian $H_{\text {int }}(\xi)$ is also dependent on components of the transverse displacement electric field operator and magnetic field operators, $d_{i}{ }^{\perp}(\xi)$ and $b_{i}(\xi)$, whose mode expansions are written as follows;

$$
\begin{aligned}
& \mathbf{d}^{\perp}\left(\mathbf{R}_{\xi}\right)=i \sum_{\mathbf{k}, \lambda}\left(\frac{\hbar c k \varepsilon_{0}}{2 V}\right)^{1 / 2}\left[\mathbf{e}_{\mathbf{k}}^{(\lambda)} a_{\mathbf{k}}^{(\lambda)} \mathrm{e}^{i \mathbf{k} \cdot \mathbf{R}_{\xi}}-\overline{\mathbf{e}}_{\mathbf{k}}^{(\lambda)} a_{\mathbf{k}}^{\dagger(\lambda)} \mathrm{e}^{-i \mathbf{k} \cdot \mathbf{R}_{\xi}}\right], \\
& \mathbf{b}\left(\mathbf{R}_{\xi}\right)=i \sum_{\mathbf{k}, \lambda}\left(\frac{\hbar k}{2 \varepsilon_{0} c V}\right)^{1 / 2}\left[\mathbf{b}_{\mathbf{k}}^{(\lambda)} a_{\mathbf{k}}^{(\lambda)} \mathrm{e}^{i \mathbf{k} \cdot \mathbf{R}_{\xi}}-\overline{\mathbf{b}}_{\mathbf{k}}^{(\lambda)} a_{\mathbf{k}}^{\dagger(\lambda)} \mathrm{e}^{-i \mathbf{k} \cdot \mathbf{R}_{\xi}}\right]
\end{aligned}
$$

where $\boldsymbol{e}_{\mathbf{k}}{ }^{(\lambda)}$ and $\boldsymbol{b}_{\mathbf{k}}{ }^{(\lambda)}$ are the generalised electric and magnetic polarization unit vectors, respectively, and $V$ is an arbitrary quantization volume defined as containing $n$ number of photons. For a mode $(\mathbf{k}, \lambda), a_{\mathbf{k}}^{(\lambda)}$ and $a_{\mathbf{k}}^{\dagger \dagger(\lambda)}$ are photon annihilation and creation operators, respectively. For non-chiral systems the electric 
dipole coupling term in equation (2) is the only term that is required in the calculation, as higher order multipole transitions feature only in very insignificant correction terms. However, in chiral systems, the low symmetry permits many transitions to be allowed by not only electric dipole selection rules, but also magnetic dipole, electric quadrupole, and higher order forms of coupling. Whilst the electric-electric and magnetic-magnetic coupling contributions are non-discriminatory, the electric-magnetic terms are discriminatory in light scattering processes.[46]

The traditional method for carrying out QED calculations is diagrammatic time-dependent perturbation theory, where Feynman diagrams[47] are commonly used as an aid in calculating the matrix element for the phenomenon being studied. A number of techniques have now been developed which give further physical insight and help circumvent the computational effort required using Feynman's time-ordered diagrams - such as state-sequence diagrams[48], response theory[49, 50], the retarded coupling approach [51], effective interaction 'collapsed vertex' Hamiltonian (by a suitable canonical transformation of the multipolar Hamiltonian) [52-54], and the induced moments method [50, 55]. In this paper it is the latter technique listed that will be the focus of interest, due to its relative computational simplicity and added physical insight. The method of induced moments is underpinned by the fact that incident electromagnetic fields induce, in polarizable bodies, moments that interact via the resonant multipole-multipole coupling tensor.[56] For example, the $\left(i_{1} \ldots i_{n}\right)$ component of the $n$ th-order electric multipole moment induced by an incident electric displacement field in a polarizable body $\xi$ is given by;

$$
P_{i_{1} \ldots i_{n}}^{(n) i n d}(\xi)=\varepsilon_{0}^{-1} \Xi_{(e) i_{1} \ldots i_{n} j_{1} \ldots j_{n}}^{(n)}(\xi, \omega) \nabla_{j_{2}} \cdots \nabla_{j_{n}} d_{j_{1}}^{\perp}\left(R_{\xi}\right)
$$

whilst the $n$ th-order magnetic multipole moment induced by a magnetic field in a polarizable body $\xi$ is:

$$
M_{i_{1} \ldots i_{n}}^{(n) i n d}(\xi)=\Xi_{(m) i_{1} \ldots i_{n} j_{1} \ldots j_{n}}^{(n)}(\xi, \omega) \nabla_{j_{2}} \cdots \nabla_{j_{n}} b_{j_{1}}\left(R_{\xi}\right)
$$

A generalized, frequency-dependent molecular polarizability $\Xi_{(e)_{1} \ldots i_{p} j_{1} \ldots j_{q}}^{(p: q)}(\xi, \omega)$ is now introduced, defined as;

$$
\Xi_{(e)_{1} \ldots i_{p} j_{1} \ldots j_{q}}^{(p: q)}(\xi, \omega)=\sum_{r}\left(\frac{\left[P_{i_{1} \ldots i_{p}}^{(p)}(\xi)\right]^{0 r}\left[P_{j_{1} \ldots j_{q}}^{(q)}(\xi)\right]^{r 0}}{\left(E_{r 0}^{\xi}-\hbar \omega\right)}+\frac{\left[P_{j_{1} \ldots j_{q}}^{(q)}(\xi)\right]^{0 r}\left[P_{i_{1} \ldots i_{p}}^{(p)}(\xi)\right]^{r 0}}{\left(E_{r 0}^{\xi}+\hbar \omega\right)}\right),
$$

where $\left[P_{i_{1} \ldots i_{n}}^{(n)}(\xi)\right]^{b a}$ are components of the $n^{\text {th }}$-order transition electric multipoles (En) for the transition from $b \leftarrow a$. The corresponding counterpart expression for a molecular polarizability cast in terms of magnetic multipoles Mn for $\Xi_{(m) i_{1} \ldots i_{p} j_{1} \ldots j_{q}}^{(p: q)}(\xi, \omega)$ has a form analogous to (7).

The above considerations are adequate for working with any order of electric multipoles, and for the present application involving mixed electric and magnetic dipoles this method is entirely suitable. An energy shift is now obtained by taking the expectation value of the appropriate matrix element for a radiation field containing $n$ photons, with both molecules assumed to be in the electronic ground state. (In fact, if the number of input photons is taken as zero, corresponding to the vacuum field, the resulting energy shift 
proves to be the dispersion force.[55] Using this method, a general expression for dispersion forces was recently obtained $[57,58]$ for molecules with arbitrary electric multipole characteristics, for both ground and excited electronic states. Nonetheless if a mixture of higher order electric and magnetic multipoles were to be examined, then a different approach would be required.) In the case of optical binding, the expectation value over some number state of the radiation field delivers an energy shift which refers to the radiation-induced change in intermolecular interaction energy.

\section{RESULTS}

\section{A. General derivation of laser-induced energy for a pair of chiral particles}

Consider two neutral polarizable chiral molecules $(A, B)$ in mutual interaction. For any such species, both the electric dipole polarizability $\alpha_{i j}(\omega)$ and its mixed electric-magnetic counterpart $G_{i j}(\omega)$ will have nonzero components. The two tensors are explicitly given by the following equations:

$$
\begin{aligned}
& \alpha_{i j}(\omega)=\sum_{r}\left\{\frac{\mu_{i}^{0 r} \mu_{j}^{r 0}}{E_{r 0}-\hbar \omega}+\frac{\mu_{j}^{0 r} \mu_{i}^{r 0}}{E_{r 0}+\hbar \omega}\right\}, \\
& G_{i j}(\omega)=\sum_{r}\left\{\frac{\mu_{i}^{0 r} m_{j}^{r 0}}{E_{r 0}-\hbar \omega}+\frac{m_{j}^{0 r} \mu_{i}^{r 0}}{E_{r 0}+\hbar \omega}\right\} .
\end{aligned}
$$

The former, which represents the simplest (lowest order) implementation of equation (7), signifies a nondiscriminatory E1 ${ }^{2}$ interaction, whereas its E1M1 analogue, $G_{i j}(\omega)$, changes sign when one enantiomer is replaced by another of opposite handedness. For later reference, it is worth noting that the tensor $\alpha_{i j}(\omega)$ is a real quantity, whereas $G_{i j}(\omega)$ is imaginary (provided the optical frequency is well away from any optical absorption band, and the basis wavefunctions are real). The underlying reason is that the E1 operator is based on charge displacement, whereas M1 entails charge circulation, through an angular momentum with an imaginary operator. It is also important to note at this stage that the formalism we have developed, leading to results that will be cast in terms of the molecular tensors $\alpha_{i j}(\omega)$ and $G_{i j}(\omega)$, is amenable to an extension to larger, chiral particles such as dielectric nanoparticles that are either intrinsically chiral, or others which are surface-functionalised with chiral species.[59] In the former case the corresponding material response tensors will be the E1 $1^{2}$ and E1M1 linear optical susceptibilities; in the latter case the effective E1M1 response tensor can be constructed as a sum of contributions from each chiral constituent. It has been shown in earlier work that there is a detailed theoretical support for such applications to optical binding between mescoscopic particles.[60]

To continue: through involvement of the $G_{i j}(\omega)$ tensor, a chiral molecule $\xi$ at position $\mathbf{R}_{\xi}$ experiences in a radiation field the induction of both an electric (10) and a magnetic (11) dipole moment, as well as an induced electric dipole moment (12) due to the $\alpha_{i j}(\omega)$ tensor: 


$$
\begin{aligned}
& \mu_{i}^{\operatorname{ind}(G)}(\xi)=G_{i j}(\xi, \omega) b_{j}\left(\mathbf{R}_{\xi}\right), \\
& m_{j}^{\operatorname{ind}(G)}(\xi)=\varepsilon_{0}^{-1} G_{i j}(\xi, \omega) d_{i}^{\perp}\left(\mathbf{R}_{\xi}\right), \\
& \mu_{i}^{\operatorname{ind}(\alpha)}(\xi)=\varepsilon_{0}^{-1} \alpha_{i j}(\xi, \omega) d_{j}^{\perp}\left(\mathbf{R}_{\xi}\right) .
\end{aligned}
$$

When the incident radiation field consists of $n$ photons of a radiation mode $(\mathbf{k}, \lambda)$, we can assume that the annihilation and (re)creation of the photons occurs in the same mode (since the emission is then stimulated, and therefore the most favoured), and the energy shift is given as the expectation value of the following expression;

$$
\begin{aligned}
\Delta E= & {\left[\mu_{i}^{\operatorname{ind}(\alpha)}(A) \mu_{j}^{\operatorname{ind}(G)}(B)+\mu_{i}^{\operatorname{ind}(G)}(A) \mu_{j}^{\operatorname{ind}(\alpha)}(B)\right] \operatorname{Re} V_{i j}(k, \mathbf{R}) } \\
& +\left[\mu_{i}^{\operatorname{ind}(\alpha)}(A) m_{j}^{\operatorname{ind}(G)}(B)+m_{i}^{\operatorname{ind}(G)}(A) \mu_{j}^{\operatorname{ind}(\alpha)}(B)\right] \operatorname{Im} U_{i j}(k, \mathbf{R}),
\end{aligned}
$$

where $\mathbf{R}$ is the inter-particle separation vector $\mathbf{R}=\mathbf{R}_{\mathrm{B}}-\mathbf{R}_{\mathrm{A}}$. Evidently, from equation (13), electric dipoles induced at each centre couple through the tensor $V_{i j}(k, \mathbf{R})$, while an electric dipole induced at one molecule and a magnetic dipole induced at the other couple through the tensor $U_{i j}(k, \mathbf{R})$ :

$$
\begin{gathered}
V_{i j}^{ \pm}(k, \mathbf{R})=\frac{1}{4 \pi \varepsilon_{0} R^{3}}\left[\left(\delta_{i j}-3 \hat{R}_{i} \hat{R}_{j}\right)(1 \pm i k R)-\left(\delta_{i j}-\hat{R}_{i} \hat{R}_{j}\right) k^{2} R^{2}\right] \mathrm{e}^{\mp i k R}, \\
U_{i j}^{ \pm}(k, \mathbf{R})=\frac{1}{4 \pi \varepsilon_{0} c R^{3}} \varepsilon_{i j k} \hat{R}_{k}\left(i k R \mp k^{2} R^{2}\right) \mathrm{e}^{\mp i k R} .
\end{gathered}
$$

The above tensors (14) and (15) are known as the retarded resonant dipole-dipole interaction tensors[45], derived in the QED representation through a calculation cast in terms of virtual photon[61] creation and annihilation events. Extending this approach, a generalised retarded resonant multipole-multipole interaction tensor has more recently been derived.[56] Substituting for the induced moments from equations (10), (11), and (12) in equation (13) produces a form of the energy shift that is cast in terms of the radiation field and the electric dipole, and mixed electric-magnetic dipole, polarizabilities of each chiral molecule (8) and (9). Hence we obtain:

$$
\begin{aligned}
\Delta E=\varepsilon_{0}^{-1} & {\left[\alpha_{i k}(A, \omega) G_{l j}^{\prime}(B, \omega) d_{k}^{\perp}\left(\mathbf{R}_{A}\right) b_{l}\left(\mathbf{R}_{B}\right)+G_{k i}(A, \omega) \alpha_{l j}(B, \omega) b_{k}\left(\mathbf{R}_{A}\right) d_{l}^{\perp}\left(\mathbf{R}_{B}\right)\right] } \\
& \times \operatorname{Re} V_{i j}(k, \mathbf{R})+\varepsilon_{0}^{-2}\left[\alpha_{i k}(A, \omega) G_{j l}(B, \omega)+G_{i k}^{\prime}(A, \omega) \alpha_{l j}(B, \omega)\right] \\
& \times d_{k}^{\perp}\left(\mathbf{R}_{A}\right) d_{l}^{\perp}\left(\mathbf{R}_{B}\right) \operatorname{Im} U_{i j}(k, \mathbf{R}) .
\end{aligned}
$$


Note that we have introduced the identity $-G_{i j}=G_{j i}^{\prime}$, which manifests itself through the fact, due to the time-odd character of the magnetic dipole operator, magnetic transition dipoles satisfy the relation $-m_{i}^{r 0}=m_{i}^{0 r}$ in the mixed electric-magnetic dipole polarizability tensor (9). The general form of the result (16) can thus be understood as coupling the polarizability tensor $\alpha$ of one particle with the $\boldsymbol{G}$ tensor of the other, which we may for shorthand denote as ' $\alpha-G$ ' coupling.

\section{B. Molecular orientation averaging and polarization analysis}

Highly distinctive kinds of polarization dependence can be anticipated to arise in systems where the two trapped particles are randomly oriented. To identify such behaviour, we must address the appropriate orientational averaging. Focusing for the present on the first term of equation (16), which we label $\mu \mu \mu m$ as it corresponds to the time-ordered diagram in Figure 1a, we first calculate its expectation value for the system state $\left|E_{0}^{A}, E_{0}^{B} ; n(\mathbf{k}, \lambda)\right\rangle$. The molecular part results in the ground state electric dipole dynamic polarizability for $A$, and the ground state mixed electric-magnetic dipole dynamic polarizability for $B$. For the electromagnetic field part, making use of the Maxwell field operators (3) and (4), the expectation value over the radiation field containing $n$ photons is:

$$
\begin{aligned}
& \left\langle n(\mathbf{k}, \lambda)\left|d_{k}^{\perp}\left(\mathbf{R}_{A}\right) b_{l}\left(\mathbf{R}_{B}\right)\right| n(\mathbf{k}, \lambda)\right\rangle \\
& =\left(\frac{\hbar k}{2 V}\right)\left[(n+1) e_{k}^{(\lambda)}(\mathbf{k}) \bar{b}_{l}^{(\lambda)}(\mathbf{k}) \mathrm{e}^{-i \mathbf{k} \cdot \mathbf{R}}+n \bar{e}_{k}^{(\lambda)}(\mathbf{k}) b_{l}^{(\lambda)}(\mathbf{k}) \mathrm{e}^{i \mathbf{k} \cdot \mathbf{R}}\right] .
\end{aligned}
$$

In equation (17), the numerical factors $(n+1)$ and $n$ in the two terms reflect a difference in origin, between contributions in which the throughput photon creation features before, and after, the corresponding photon annihilation. On assumption that the mode occupation number is sufficiently large for $n+1 \sim n$, the two terms in (17) can be recognized as complex conjugates, allowing twice the real part to be taken. Thus, the energy shift can be expressed more concisely as:

$$
\Delta E_{\mu \mu \mu m}=\frac{n \hbar k}{\varepsilon_{0} V} \alpha_{i k}(A, \omega) G_{l j}^{\prime}(B, \omega) e_{k}^{(\lambda)}(\mathbf{k}) \bar{b}_{l}^{(\lambda)}(\mathbf{k}) \operatorname{Re} V_{i j}(k, \mathbf{R}) \cos (\mathbf{k} \cdot \mathbf{R})
$$

Using standard techniques[62], an orientational average is now performed on equation (18) to deliver results for the interactions of molecules whose relative displacement $\mathbf{R}$ is fixed in position with respect to the beam of throughput radiation, but whose individual orientations are random and uncorrelated;

$$
\left\langle\alpha_{i k}(A, \omega) G_{l j}^{\prime}(B, \omega)\right\rangle=\frac{1}{9} \delta_{i k} \delta_{l j} \alpha_{\lambda \mu}(A, \omega) G_{\alpha \beta}^{\prime}(B, \omega) \delta_{\lambda \mu} \delta_{\alpha \beta},
$$

where $\delta_{i j}$ is the Kronecker delta, symmetric in its indices. Equation (19) leads to the following energy shift; 


$$
\Delta E_{\mu \mu \mu m}=\frac{n \hbar k}{\varepsilon_{0} V} \alpha(A, \omega) G^{\prime}(B, \omega) e_{i}^{(\lambda)}(\mathbf{k}) \bar{b}_{j}^{(\lambda)}(\mathbf{k}) \operatorname{Re} V_{i j}(k, \mathbf{R}) \cos (\mathbf{k} \cdot \mathbf{R}),
$$

using a simplified notation for scalars derived from the tensor traces, $\alpha(\xi, \omega)=\frac{1}{3} \alpha_{\lambda \lambda}(\xi, \omega)$ and $G^{\prime}(\xi, \omega)=\frac{1}{3} G_{\alpha \alpha}^{\prime}(\xi, \omega)$. Carrying out a similar analysis on the other three terms of equation (16) yields the total energy shift as:

$$
\begin{aligned}
\Delta E=\frac{n \hbar k}{\varepsilon_{0} V}\{ & {\left[\alpha(A, \omega) G^{\prime}(B, \omega) e_{i}^{(\lambda)}(\mathbf{k}) \bar{b}_{j}^{(\lambda)}(\mathbf{k})+G(A, \omega) \alpha(B, \omega) b_{i}^{(\lambda)}(\mathbf{k}) \bar{e}_{j}^{(\lambda)}(\mathbf{k})\right] } \\
& \times \operatorname{Re} V_{i j}(k, \mathbf{R})+c\left[\alpha(A, \omega) G(B, \omega)+G^{\prime}(A, \omega) \alpha(B, \omega)\right] e_{i}^{(\lambda)}(\mathbf{k}) \bar{e}_{j}^{(\lambda)}(\mathbf{k}) \\
& \left.\times \operatorname{Im} U_{i j}(k, \mathbf{R})\right\} \cos (\mathbf{k} \cdot \mathbf{R}) .
\end{aligned}
$$

At this stage, a key distinction emerges between the behaviour that can be observed with plane and with circularly polarised light. For the pair of molecules in a positionally fixed configuration, the molecular orientationally averaged result (21) for the radiation-induced total energy is zero when the incident light is linearly polarized, regardless of whether the wave-vector of the light is parallel or perpendicular to $\mathbf{R}$. This is evident after expanding both $\operatorname{Re} V_{i j}(k, \mathbf{R})$ and $\operatorname{Im} U_{i j}(k, \mathbf{R})$, and using relations based on the transversality and real character of the plane polarization vectors: $\delta_{i j} e_{i}^{(\lambda)}(\mathbf{k}) \bar{b}_{j}^{(\lambda)}(\mathbf{k})=0, \hat{R}_{i} \hat{R}_{j} e_{i}^{(\lambda)}(\mathbf{k}) \bar{b}_{j}^{(\lambda)}(\mathbf{k})=0$, and $\hat{R}_{k} \varepsilon_{i j k} e_{i}^{(\lambda)}(\mathbf{k}) \bar{e}_{j}^{(\lambda)}(\mathbf{k})=0$. However, the use of circularly polarized incident light results in non-zero energies for both $\mathbf{k} \| \mathbf{R}$ and $\mathbf{k} \perp \mathbf{R}$.

Assuming a circular polarization, therefore, we now process the first term of equation (21), expanding $\operatorname{Re} V_{i j}(k, \mathbf{R})$, and contracting the tensor components with the aid of the following relation;

$$
e_{i}^{(\mathrm{L} / \mathrm{R})}(\mathbf{k}) \bar{b}_{j}^{(\mathrm{L} / \mathrm{R})}(\mathbf{k})= \pm i e_{i}^{(\mathrm{L} / \mathrm{R})}(\mathbf{k}) \bar{e}_{j}^{(\mathrm{L} / \mathrm{R})}(\mathbf{k})=\frac{1}{2}\left[ \pm i\left(\delta_{i j}-\hat{k}_{i} \hat{k}_{j}\right)+\varepsilon_{i j k} \hat{k}_{k}\right]
$$

with $\varepsilon_{i j k}$ representing the antisymmetric Levi-Civita tensor in three dimensions. Only the first term in square brackets of equation (22) contributes since $\operatorname{Re} V_{i j}(k, \mathbf{R})$ is $i j$-symmetric, and for $\mathbf{k} \perp \mathbf{R}$ the energy shift is therefore seen to be;

$$
\Delta E_{\mu \mu \mu m}^{\perp(\mathrm{L} / \mathrm{R})}=\operatorname{Re} \frac{I}{8 \pi \varepsilon_{0}^{2} c^{2} R^{3}} \alpha(A, \omega) G^{\prime}(B, \omega)\left[(\mp i)(\cos k R+k R \sin k R) \mp i k^{2} R^{2} \cos k R\right],
$$

where $I$ is the input laser irradiance and is equal to $\left(n \hbar c^{2} k / V\right)$. A similar analysis for the remaining three terms in equation (21) for two neutral chiral molecules in the presence of a circularly polarised beam propagating $\mathbf{k} \perp \mathbf{R}$ gives the overall energy shift as; 


$$
\begin{aligned}
\Delta E^{\perp(\mathrm{L} / \mathrm{R})}=\frac{I}{8 \pi \varepsilon_{0}^{2} c^{2} R^{3}}\left\{\left(\tilde{G}(A, \omega) \alpha(B, \omega)-\alpha(A, \omega) \tilde{G}^{\prime}(B, \omega)\right)\right. & \\
\times & {\left.\left[ \pm(\cos k R+k R \sin k R) \pm k^{2} R^{2} \cos k R\right]\right\}, }
\end{aligned}
$$

where we have further simplified the notation using $\tilde{G}^{\prime}(\xi, \omega)=i G^{\prime}(\xi, \omega)$. The 'Re', which signifies to take the real part of the equation, has been dropped from equation (24) as no imaginary parts longer exist, due to the tensor $G$ itself being imaginary, as noted earlier. For circularly polarised light propagating parallel $\mathbf{k} \| \mathbf{R}$, the total energy shift for a pair of neutral chiral molecules finally emerges as:

$$
\begin{aligned}
\Delta E^{\|(\mathrm{L} / \mathrm{R})}=\frac{I}{4 \pi \varepsilon_{0}^{2} c^{2} R^{3}}\{( & \left.\tilde{G}(A, \omega) \alpha(B, \omega)-\alpha(A, \omega) \tilde{G}^{\prime}(B, \omega)\right) \\
\times & {\left.\left[\mp(\cos k R+k R \sin k R) \pm k^{2} R^{2} \cos k R\right]\right\} \cos (k R) . }
\end{aligned}
$$

\section{Phased-weighted pair orientational average analysis}

The next step is to carry out a pair orientational average, pertaining to a common situation where the molecular pair are in a gaseous or liquid state. This involves averaging over all $\mathbf{k}$ relative to $\mathbf{R}$, and over the relative orientations of the pair of molecules in the system. As we are concerned with a pair interaction with radiation, we require the use of a phased rotational average, accounting for the difference in optical phase of the throughput radiation at each center.[63] In the present case, the relevant phased rotational average takes the generic form;

$$
\Delta E^{\phi}=K \operatorname{Re}\left\langle S_{i_{1} i_{2}}(\mathbf{k}) T_{\lambda_{1} \lambda_{2}}(\mathbf{R}) e^{i \mathbf{k} \cdot \mathbf{R}}\right\rangle
$$

where $S_{i_{1} i_{2}}(\mathbf{k})$ and $\mathbf{k}$ are respectively tensors and vectors which are fixed in a laboratory frame of reference; in our specific example $\mathbf{k}$ relates to the wave vector of light and $S_{i_{1} i_{2}}(\mathbf{k})$ the two polarization vectors: $T_{\lambda_{1} \lambda_{2}}(\mathbf{R})$ and $\mathbf{R}$ are tensors and vectors fixed in a molecular frame, with $T_{\lambda_{1} \lambda_{2}}(\mathbf{R})$ the appropriate retarded resonant dipole-dipole interaction tensor as given by (14) and (15); $K$ is a constant. The above expression can then be cast as;

$$
I_{i_{1}, \ldots, i_{m}: \lambda_{1}, \ldots, \lambda_{m}}^{(m),}(\alpha, \hat{k}, \hat{R}) \equiv\left\langle l_{i_{1} \lambda_{1}} \cdots l_{i_{m} \lambda_{m}} e^{i \alpha \hat{k} \cdot \hat{R}}\right\rangle,
$$


where $\alpha=|\mathbf{k}||\mathbf{R}|, m$ is the rank of the tensor to be averaged, and $l_{i_{p} \lambda_{p}}$ is the direction cosine of the angle between the laboratory axis $i_{p}$ and the molecular axis $\lambda_{p . .}$ The following results for $I_{i_{1}, \ldots, i_{m}: \lambda_{1}, \ldots, \lambda_{m}}^{(m)}(\alpha, \hat{u}, \hat{w})$, defined by equation (27), are expressed in terms of spherical Bessel functions $j_{m}(\alpha)$ [64] for $m \leq 2$ :

$$
\begin{aligned}
& \underline{m=0} \\
& I^{(0) \phi}(\alpha, \hat{k}, \hat{R})=j_{0}(\alpha) \\
& m=2
\end{aligned}
$$

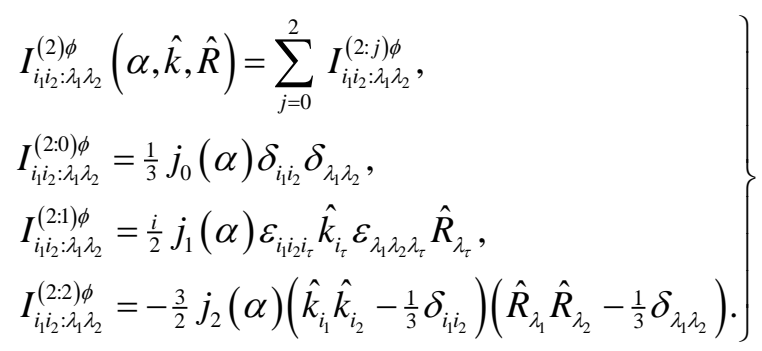

Just as with the results of the previous sub-section, the energy shift for a pair of freely tumbling chiral molecules in the presence of linear polarised light is zero, but it becomes non-zero when circularly polarised light is used. Taking the first term of equation (21) once again and expanding $\operatorname{Re} V_{i j}(k, \mathbf{R})$, the energy shift that requires phased averaging is of the form:

$$
\begin{array}{r}
\left\langle\Delta E_{\mu \mu \mu m}\right\rangle=\operatorname{Re}\left(\frac{I}{4 \pi \varepsilon_{0}^{2} c^{2} R^{3}}\right) \alpha(A, \omega) G^{\prime}(B, \omega)\left\{\left\langle e_{i}^{(\mathrm{L} / \mathrm{R})}(\mathbf{k}) \bar{b}_{j}^{(\mathrm{L} / \mathrm{R})}(\mathbf{k})\left(\delta_{i j}-3 \hat{R}_{i} \hat{R}_{j}\right)(\cos k R+k R \sin k R) e^{i \mathbf{k} \cdot \mathbf{R}}\right\rangle\right. \\
\left.-\left\langle e_{i}^{(\mathrm{L} / \mathrm{R})}(\mathbf{k}) \bar{b}_{j}^{(\mathrm{L} / \mathrm{R})}(\mathbf{k})\left(\delta_{i j}-\hat{R}_{i} \hat{R}_{j}\right)\left(k^{2} R^{2} \cos k R\right) e^{i \mathbf{k} \cdot \mathbf{R}}\right)\right\} .
\end{array}
$$

Taking the first term in curly brackets of equation (30) and expanding;

$$
\left\langle e_{i}^{(\mathrm{L} / \mathrm{R})}(\mathbf{k}) \bar{b}_{j}^{(\mathrm{L} / \mathrm{R})}(\mathbf{k}) \delta_{i j}(\cos k R+k R \sin k R) e^{i \mathbf{k} \cdot \mathbf{R}}\right\rangle-\left\langle e_{i}^{(\mathrm{L} / \mathrm{R})}(\mathbf{k}) \bar{b}_{j}^{(\mathrm{L} / \mathrm{R})}(\mathbf{k}) 3 \hat{R}_{i} \hat{R}_{j}(\cos k R+k R \sin k R) e^{i \mathbf{k} \cdot \mathbf{R}}\right\rangle,
$$

using $\bar{b}_{j}^{(\mathrm{L} / \mathrm{R})}(\mathbf{k})= \pm i \bar{e}_{j}^{(\mathrm{L} / \mathrm{R})}(\mathbf{k})$ and $e_{i}^{(\mathrm{L} / \mathrm{R})}(\mathbf{k}) \bar{e}_{j}^{(\mathrm{L} / \mathrm{R})}(\mathbf{k})=1$ and $m=0$ from (28), the phased average in the first term in angular brackets of (31) reduces to:

$$
\left\langle \pm i e^{i \mathbf{k} \cdot \mathbf{R}}\right\rangle= \pm i j_{0}(k R)
$$


For the second term in angular brackets of (31) we use $\bar{b}_{j}^{(\mathrm{L} / \mathrm{R})}(\mathbf{k})= \pm i \bar{e}_{j}^{(\mathrm{L} / \mathrm{R})}(\mathbf{k})$ again and the $m=2$ result from (29). Taking this term by term:

$$
\begin{gathered}
3 \pm i \hat{R}_{\lambda} \hat{R}_{\mu} e_{i}^{(\mathrm{L} / \mathrm{R})}(\mathbf{k}) \bar{e}_{j}^{(\mathrm{L} / \mathrm{R})}(\mathbf{k}) \frac{1}{3} j_{0}(k R) \delta_{i j} \delta_{\lambda \mu}= \pm i j_{0}(k R), \\
\pm 3 i \hat{R}_{\lambda} \hat{R}_{\mu} e_{i}^{(\mathrm{L} / \mathrm{R})}(\mathbf{k}) \bar{e}_{j}^{(\mathrm{L} / \mathrm{R})}(\mathbf{k}) \frac{i}{2} j_{1}(k R) \varepsilon_{i j k} \hat{k}_{\lambda} \varepsilon_{\lambda \mu v} \hat{R}_{v}=0, \\
\mp \frac{9}{2} i \hat{R}_{\lambda} \hat{R}_{\mu} e_{i}^{(\mathrm{L} / \mathrm{R})}(\mathbf{k}) \bar{e}_{j}^{(\mathrm{L} / \mathrm{R})}(\mathbf{k}) j_{2}(k R)\left[\hat{k}_{i} \hat{k}_{j}-\frac{1}{3} \delta_{i j}\right]\left[\hat{R}_{\lambda} \hat{R}_{\mu}-\frac{1}{3} \delta_{\lambda \mu}\right]= \pm i j_{2}(k R) .
\end{gathered}
$$

Therefore, the overall phased average result for equation (31) is:

$$
\left\langle e_{i}^{(\mathrm{L} / \mathrm{R})}(\mathbf{k}) \bar{b}_{j}^{(\mathrm{L} / \mathrm{R})}(\mathbf{k})\left(\delta_{i j}-3 \hat{R}_{i} \hat{R}_{j}\right)(\cos k R+k R \sin k R) e^{i \mathbf{k} \cdot \mathbf{R}}\right\rangle=\mp i j_{2}(k R)[\cos k R+k R \sin k R] .
$$

A similar analysis for the second term in curly brackets of (30) gives the result;

$$
\left\langle e_{i}^{(\mathrm{L} / \mathrm{R})}(\mathbf{k}) \bar{b}_{j}^{(\mathrm{L} / \mathrm{R})}(\mathbf{k})\left(\delta_{i j}-\hat{R}_{i} \hat{R}_{j}\right)\left(k^{2} R^{2} \cos k R\right) e^{i \mathbf{k} \cdot \mathbf{R}}\right\rangle=\left( \pm \frac{2 i}{3} j_{0}(k R) \mp \frac{i}{3} j_{2}(k R)\right)\left(k^{2} R^{2} \cos k R\right),
$$

which gives the overall phase-averaged energy shift for (30) as:

$$
\begin{array}{r}
\left\langle\Delta E_{\mu \mu \mu m}\right\rangle=\left(\frac{I}{4 \pi \varepsilon_{0}^{2} c^{2} R^{3}}\right) \alpha(A, \omega) \tilde{G}^{\prime}(B, \omega)\left\{\mp j_{2}(k R)(\cos k R+k R \sin k R)\right. \\
\left.-\left( \pm \frac{2}{3} j_{0}(k R) \mp \frac{1}{3} j_{2}(k R)\right) k^{2} R^{2} \cos k R\right\} .
\end{array}
$$

Finally, averaging the remaining three terms of equation (21), expanding the spherical Bessel functions and using double angle formulae gives the total phased-averaged energy as: 


$$
\begin{aligned}
\left\langle\Delta E^{(L / R)}\right\rangle= \pm \frac{I}{4 \pi \varepsilon_{0}^{2} c^{2} R^{3}} & \left(\alpha(A, \omega) \tilde{G}^{\prime}(B, \omega)-\tilde{G}(A, \omega) \alpha(B, \omega)\right) \\
& \times\left(\cos 2 k R-\frac{k R \sin 2 k R}{2}+\frac{3 \sin 2 k R}{2 k R}+\frac{3 \cos 2 k R}{(k R)^{2}}-\frac{3 \sin 2 k R}{2(k R)^{3}}\right) .
\end{aligned}
$$

This is the main result for the differential energy shift experienced by two neutral, randomly oriented chiral molecules $(A, B)$ bound together by the presence of circularly polarised light. As with the dominant ' $\alpha$ - $\alpha$ ' coupling, the result clearly depends linearly on the irradiance of the input beam, whilst the discriminatory effects embedded in the ' $\alpha$ - $G$ ' coupling alone arise from the handedness of the radiation and the mixed electric-magnetic polarizabilities of the molecules. Taking two chemically identical chiral particles, one left-handed and the other right-handed, it is evident that the binding energy contributions (24), (25) and (39) will be zero. It is worth noting, however, that when the chiral species A and B are not identical, their $G$ tensors will generally differ in magnitude and (24), (25) and (39) will be non-zero. The energy shifts experienced by pairs of the same molecules with the same handedness will differ, the sign of their chirally sensitive components depending on the relative handedness of the incident light and of the molecules themselves. For example, if the incident beam is of a specific handedness, the optical binding of two lefthanded molecules will differ in energy from the binding of two right-handed molecules: the effect is discriminatory. On changing the handedness of light, the signs reverse, as illustrated in Figure 2. In each instance, the usual position-dependence of intensity within the spatial profile of the laser beam will signify that the pair experience differential restoring forces for any displacements from their equilibrium configuration.

\section{DISCUSSION}

Our general analysis has focused upon a previously overlooked mechanism that operates between chiral particles held in an optical trap. In particular it elucidates the discriminatory optical binding forces that come into play in the presence of a sufficiently intense, circularly polarized laser beam. It has been revealed how the discrimination of particle chirality arises from both the polarization of the input light and the mixed electric-magnetic polarizability $G(\xi, \omega)$ of the species present. In earlier work by Salam,[40] identifying a discriminatory effect due to a ' $G-G$ 'coupling, chiral discrimination based entirely on the mixed electricmagnetic polarizability was estimated to be in the order of $10^{-6}$ smaller than the dominant ' $\alpha-\alpha$ 'coupling. In the mechanism presented in this paper, however, the discriminatory ' $\alpha$ - $G$ ' binding energies prove to be more significant - potentially a magnitude of $10^{3}$ or more times larger than in Salam's pioneering work. It is not only the difference in magnitude of the optical binding energies between the two mechanisms that proves to be of interest, since the interaction we have described draws out the interplay of material and optical chirality, affording new opportunities to exploit the effects of varying circular polarization.[65] In considering the scope for applications, it should also be borne in mind that there is a possibility of further enhancing the binding energies by exploiting pre-resonance enhancement of $G(\xi, \omega)$.

One possible exploitation of these discriminatory optical binding forces lies in the helicity-dependent manipulation of chiral particles. Another distinct application could be in optical strategies for chiral sorting. Although a host of established methods exist for chiral resolution at the molecular level[66], such methods generally rely on other materials to act as resolving agents. Other systems for chiral sorting of micro-sized objects instead are based on the different hydrodynamical forces associated with the different chiral shape for each enantiomer.[67-70] More contemporary work in the field involves the use of optomechanical forces and laser light to act as potential methods of enantiomeric separation. For example, experimental 
work has promoted the differential absorption of left- and right-circularly polarised light (circular dichroism) as a chiral sorting strategy for micro-objects in a fluidic environment.[71] It has also been shown, through an application of QED theory, that discriminatory optical trapping forces - though small can act as a mechanism to separate left- and right-handed molecules.[38, 72]

In conclusion, although it is likely to prove difficult to implement these discriminatory effects in the direct pursuit of enantiomer separation, there may be scope to develop a means of identifying chirality in optically bound systems, using conventional laser optical instrumentation. It is interesting to consider a possible experimental strategy. For example, if the input light is modulated between right and left circular polarizations, the response of a system comprising chiral particles with a common sense of handedness will be a corresponding oscillation in their equilibrium positions. In particular, if the modulation frequency is tuned into resonance with the essentially harmonic natural frequency of the optically bound pair,[13] such small-scale oscillations should become readily detectable by conventional optical methods, and so serve both to identify and characterize the chiral nature of the bound particles. The detailed exploration of such effects represents scope for future work.

\section{ACKNOWLEDGEMENTS}

We are pleased to acknowledge helpful comments from our colleague Dr David Bradshaw. Financial support in the form of a UEA research studentship to KAF is also gratefully recorded. 


\section{CAPTIONS FOR THE FIGURES}

Figure 1. (color online) Four permutations of $\mathrm{E} 1$ and $\mathrm{M} 1$ interactions in ' $\mu \mu \mu \mathrm{m}$ ' contributions to the 48 distinct Feynman time-ordered diagrams for optical binding, in one representative time-ordering.

Figure 2. (color online) Illustration of the equivalences, and non-equivalences, between the optical binding energies for chiral particles of different handedness (depicted by the dark purple and pale blue spheres) irradiated by circularly polarized light of either circularity (right- and left-handed forms shown with opposite twist). 
FIGURES
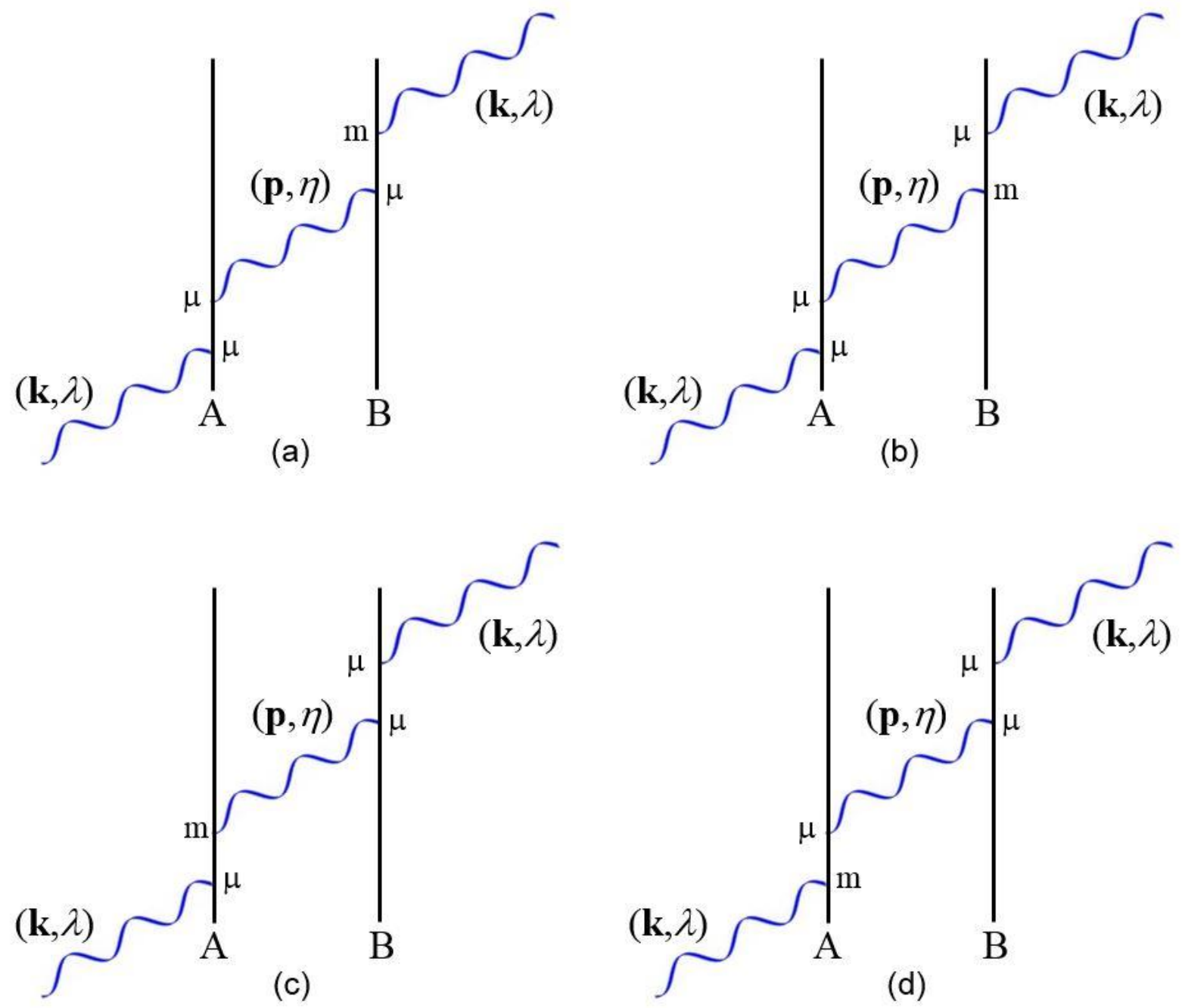

Figure 1 


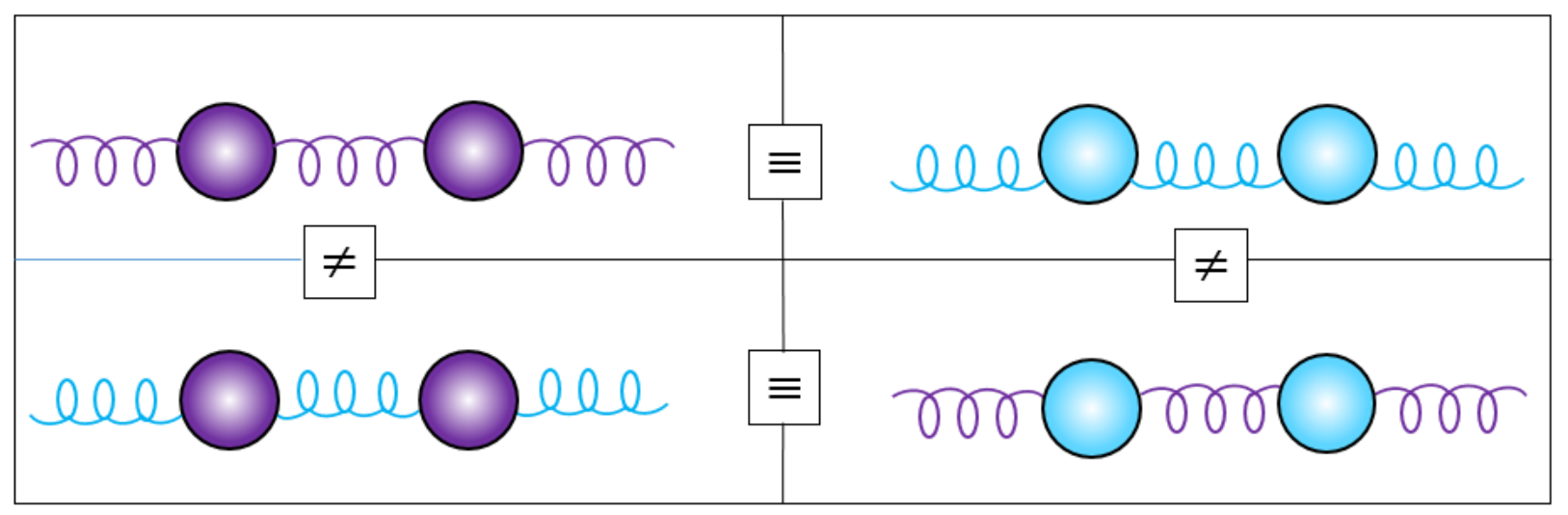

Figure 2 


\section{REFERENCES}

[1] H. B. G. Casimir and D. Polder, Phys. Rev. 73, 360 (1948).

[2] R. H. French, J. Am. Ceram. Soc. 83, 2117 (2000).

[3] T. Thirunamachandran, Mol. Phys. 40, 393 (1980).

[4] T. Čižmár, L. C. Dávila Romero, K. Dholakia, and D. L. Andrews, J. Phys. B: At. Mol. Opt. Phys. 43, 102001 (2010).

[5] K. Dholakia and P. Zemanek, Rev. Mod. Phys. 82, 1767 (2010).

[6] M. M. Burns, J.-M. Fournier, and J. A. Golovchenko, Phys. Rev. Lett. 63, 1233 (1989).

[7] A. Ashkin, J. Dziedzic, J. Bjorkholm, and S. Chu, Opt. Lett. 11, 288 (1986).

[8] G. Whitesides, Angew. Chem. Int. Ed. (English) 29, 1209 (1990).

[9] S. A. Tatarkova, A. E. Carruthers, and K. Dholakia, Phys. Rev. Lett. 89, 283901 (2002).

[10] W. Singer, M. Frick, S. Bernet, and M. Ritsch-Marte, JOSA B 20, 1568 (2003).

[11] D. M. Gherardi, A. E. Carruthers, T. Čižmár, E. M. Wright, and K. Dholakia, Appl. Phys. Lett. 93, 041110 (2008).

[12] N. Metzger, K. Dholakia, and E. Wright, Phys. Rev. Lett. 96, 068102 (2006).

[13] N. Metzger, R. Marchington, M. Mazilu, R. Smith, K. Dholakia, and E. Wright, Phys. Rev. Lett. 98, 068102 (2007).

[14] N. Metzger, E. Wright, and K. Dholakia, New J. Phys. 8, 139 (2006).

[15] V. Karásek, T. Čižmár, O. Brzobohatý, P. Zemánek, V. Garcés-Chávez, and K. Dholakia, Phys. Rev. Lett. 101, 143601 (2008).

[16] V. Karásek, K. Dholakia, and P. Zemánek, Appl. Phys. B 84, 149 (2006).

[17] V. Karásek, O. Brzobohatý, and P. Zemánek, Journal of Optics A: Pure and Applied Optics 11, 034009 (2009).

[18] D. S. Bradshaw and D. L. Andrews, Phys. Rev. A 72, 033816 (2005).

[19] L. D. Romero and D. L. Andrews, Chapter 4 - Nanoscale Optics: Interparticle Forces. in D. L. Andrews (Ed.) Structured Light and Its Applications: An Introduction to Phase-Structured Beams and Nanoscale Optical Forces (Academic, Amsterdam, Boston, 2008), (pp. 79-115).

[20] A. Salam, Phys. Rev. A 73, 013406 (2006).

[21] D. L. Andrews and D. S. Bradshaw, Opt. Lett. 30, 783 (2005).

[22] J. Rodríguez, L. C. Dávila Romero, and D. L. Andrews, J. Nanophoton. 1, 019503 (2007).

[23] J. Rodríguez, L. C. Dávila Romero, and D. L. Andrews, Phys. Rev. A 78, 043805 (2008).

[24] L. C. Dávila Romero, J. Rodríguez, and D. L. Andrews, Opt. Commun. 281, 865 (2008).

[25] D. S. Bradshaw, K. A. Forbes, and D. L. Andrews, Proc. SPIE, 91260P (2014).

[26] J. Rodríguez and D. L. Andrews, Opt. Commun. 282, 2267 (2009).

[27] D. L. Andrews and J. Rodríguez, Opt. Lett. 33, 1830 (2008).

[28] D. S. Bradshaw and D. L. Andrews, Proc. SPIE 5736, 87 (2005).

[29] J. Rodríguez and D. L. Andrews, Opt. Lett. 33, 2464 (2008).

[30] D. L. Andrews, Structured Light and its Applications: An Introduction to Phase-Structured Beams and Nanoscale Optical Forces (Academic, Amsterdam, Boston, 2008), p. 2.

[31] V. Demergis and E.-L. Florin, Nano Lett. 12, 5756 (2012).

[32] Z. Yan, S. K. Gray, and N. F. Scherer, Nat. Commun. 5 (2014).

[33] D. P. Craig, E. A. Power, and T. Thirunamachandran, Proc. R. Soc. A 322, 165 (1971).

[34] D. P. Craig and D. P. Mellor, in Bonding Structure (Springer, 1976), pp. 1.

[35] D. Craig and T. Thirunamachandran, J. Chem. Phys. 109, 1259 (1998).

[36] E. A. Power and T. Thirunamachandran, J. Chem. Phys. 60, 3695 (1974).

[37] L. D. Barron and A. D. Buckingham, Mol. Phys. 20, 1111 (1971).

[38] D. S. Bradshaw and D. L. Andrews, New J. Phys. 16, 103021 (2014).

[39] G. Tkachenko and E. Brasselet, Nat. Commun. 5, 4491 (2014).

[40] A. Salam, J. Chem. Phys. 124, 014302 (2006).

[41] R. G. Woolley, Proc. R. Soc. A 456, 1803 (2000). 
[42] E. A. Power and S. Zienau, Phil. Trans. R. Soc. Lond. A 251, 427 (1959).

[43] R. Woolley, Proc. R. Soc. A 321, 557 (1971).

[44] E. A. Power and T. Thirunamachandran, Am. J. Phys. 46, 370 (1978).

[45] D. P. Craig and T. Thirunamachandran, Molecular Quantum Electrodynamics: An Introduction to Radiation-Molecule Interactions (Dover Publications, Mineola, NY, 1998).

[46] L. D. Barron, Molecular Light Scattering and Optical Activity (Cambridge University Press Cambridge, 2004), Vol. 2.

[47] R. P. Feynman, Phys. Rev. 76, 769 (1949).

[48] R. D. Jenkins and D. L. Andrews, J. Chem. Phys. 116, 6713 (2002).

[49] E. A. Power and T. Thirunamachandran, Phys. Rev. A 28, 2671 (1983).

[50] D. Craig and T. Thirunamachandran, Theor. Chem. Acc. 102, 112 (1999).

[51] L. C. Dávila Romero and D. L. Andrews, J. Phys. B: At. Mol. Opt. Phys. 42, 085403 (2009).

[52] D. Craig and E. Power, Int. J. Quant. Chem. 3, 903 (1969).

[53] D. L. Andrews and T. Thirunamachandran, Proc. R. Soc. A 358, 297 (1978).

[54] D. Craig, E. Power, and T. Thirunamachandran, Proc. R. Soc. A 348, 19 (1976).

[55] E. Power and T. Thirunamachandran, Phys. Rev. A 48, 4761 (1993).

[56] A. Salam, J. Chem. Phys 122, 044112 (2005).

[57] A. Salam, J. Phys. B: At. Mol. Opt. Phys. 39, S651 (2006).

[58] A. Salam, J. Phys. B: At. Mol. Opt. Phys. 39, S663 (2006).

[59] V. V. Klimov, I. V. Zabkov, A. A. Pavlov, and D. V. Guzatov, Opt. Express 22, 18564 (2014).

[60] J. Rodriguez, Opt. Lett. 33, 2197 (2008).

[61] D. L. Andrews and D. S. Bradshaw, Eur. J. Phys. 25, 845 (2004).

[62] D. L. Andrews and T. Thirunamachandran, J. Chem. Phys. 67, 5026 (1977).

[63] D. L. Andrews and M. J. Harlow, J. Chem. Phys. 80, 4753 (1984).

[64] K. Riley, M. Hobson, and S. Bence, Am. J. Phys. 67, 165 (1999).

[65] D. S. Bradshaw, J. M. Leeder, M. M. Coles, and D. L. Andrews, Chem. Phys. Lett. (in press)

[66] T. J. Ward and B. A. Baker, Anal. Chem. 80, 4363 (2008).

[67] R. Eichhorn, Phys. Rev. Lett. 105, 034502 (2010).

[68] M. Kostur, M. Schindler, P. Talkner, and P. Hänggi, Phys. Rev. Lett. 96, 014502 (2006).

[69] S. Meinhardt, J. Smiatek, R. Eichhorn, and F. Schmid, Phys. Rev. Lett. 108, 214504 (2012).

[70] R. Eichhorn, Chem. Phys. 375, 568 (2010).

[71] G. Tkachenko and E. Brasselet, Nat. Commun. 5, 3577 (2014).

[72] D. S. Bradshaw and D. L. Andrews, Opt. Lett. 40, 677 (2015). 